\title{
The absence of a pattern and the occurrences of another
}

\author{
Miklós Bóna \\ Dep. of Mathematics, Univ. of Florida, 358 Little Hall, PO Box 118105, Gainesville, FL 32611-8105 (USA)
}

received Jan. 10, 2010, accepted Feb. 14, 2010.

Following a question of J. Cooper, we study the expected number of occurrences of a given permutation pattern $q$ in permutations that avoid another given pattern $r$. In some cases, we find the pattern that occurs least often, (resp. most often) in all $r$-avoiding permutations. We also prove a few exact enumeration formulae, some of which are surprising.

Keywords: Permutation pattern - generating functions

\section{Introduction}

Let $q=q_{1} q_{2} \ldots q_{k}$ be a permutation in the symmetric group $S_{k}$. We say that the permutation $p=$ $p_{1} p_{2} \ldots p_{n} \in S_{n}$ contains a q-pattern if and only if there is a subsequence $p_{i_{1}} p_{i_{2}} \ldots p_{i_{k}}$ of $p$ whose elements are in the same relative order as those in $q$, that is,

$$
p_{i_{t}}<p_{i_{u}} \text { if and only if } q_{t}<q_{u}
$$

whenever $1 \leq t, u \leq k$. If $p$ does not contain $q$, then we say that $p$ avoids $q$. For example, 41523 contains exactly two occurrences of the pattern 132, namely 152 and 153 , while 34512 avoids 132 . See Chapter 14 of [1] for an introduction to pattern avoiding permutations, and Chapters 4 and 5 of [2] for a somewhat more detailed treatment. A recent collection of research articles can be found in [5].

It is straightforward to compute, using the linear property of expectation, that the average number of $q$-patterns in a randomly selected permutation of length $n$ is $\frac{1}{k !}\left(\begin{array}{l}n \\ k\end{array}\right)$, where $k$ is the length of $q$.

Joshua Cooper [4] has raised the following interesting family of questions. Let $r$ be a given permutation pattern. What can be said about the average number of occurrences of $q$ in a randomly selected $r$-avoiding permutation?

In this paper, we study this family of questions in the case when $r=132$. We prove the perhaps surprising result that among patterns of a fixed length $k$, it is the increasing pattern $12 \cdots k$ that occurs least often and it is the decreasing pattern $k(k-1) \cdots 1$ that occurs most often in a randomly selected 132avoiding permutation. While pattern avoiding permutations in general has been a very popular topic in the last fifteen years, this paper joins a rather short list of articles ([3] is an example) in which expectations of the number of occurrences of a pattern are computed. 


\section{Preliminaries}

The structure of 132-avoiding permutations is well understood. If $p=p_{1} p_{2} \cdots p_{n}$ is such a permutation, and $p_{i}=n$, then $p_{t}>p_{u}$ must hold for all pairs $(t, u)$ satisfying $t<i<u$. In other words, all entries on the left of the entry $n$ must be larger than all entries of the right of $n$. Indeed, if this does not happen, then $p_{t} n p_{u}$ is a 132-pattern. This property is so central to the work carried out in this paper that we illustrate it by Figure 2 .

Therefore, if $C_{n}$ denotes the number of 132-avoiding permutations of length $n$, then the numbers $C_{n}$ satisfy the recurrence relation

$$
C_{n}=\sum_{i=1}^{n} C_{i-1} C_{n-i}
$$

with $C_{0}=1$. Hence the numbers $C_{n}$ are identical to the famous Catalan numbers $C_{n}=\left(\begin{array}{c}2 n \\ n\end{array}\right) /(n+1)$. See Chapter 6 of Enumerative Combinatorics [6] for a wealth of information on Catalan numbers.

It follows from the structural property described in the first paragraph of this section that a 132-avoiding permutation either ends in its largest entry, or it is decomposable, that is, it can be cut into two parts so that every entry that precedes that cut is larger than every entry that follows that cut. Indeed, if the maximal entry $n$ is not in the rightmost position, then one can cut the permutation immediately after $n$ to obtain such a cut. Note that there may be additional ways to cut the same permutation. (We mention that some authors use a different definition of decomposable permutations, one that could be called the dual of this one, but the present definition suits the purposes of this paper better.)

Example 1. The permutation 76834512 is decomposable. One possible cut is $768 \mid 34512$, and another one is $768345 \mid 12$.

This relatively simple structure of 132-avoiding permutations enables us to give an exhaustive list of the ways in which a 132-avoiding permutation can contain a given pattern.

Fact 1. If the 132-avoiding permutation $p$ of length $n$ contains a copy $Q$ of the pattern $q$ of length $k$, then one of the following holds. (Note that $q$ itself must be 132-avoiding; otherwise $p$ clearly avoids $q$.)

1. If $q$ is not decomposable, that is, if $q$ ends in its largest entry, then

(a) either all of $Q$ must be on the left of $n$,

(b) or all of $Q$ must be on the right of $n$,

(c) or $Q$ must end in $n$.

For instance, if $q=123$, then in $p=678952134$, the subsequence 678 precedes $n=9$, the subsequence 679 ends in 9, and the subsequence 134 follows 9.

2. If $q$ is decomposable, that is, when $q$ does not end in its largest entry, and $q$ does not start with its largest entry, then

(a) either all of $Q$ is on the left of $n$, 
(b) or all of $Q$ is on the right of $n$,

(c) or the part of $Q$ that precedes a given cut is on the left of $n$ and the part of $Q$ the follows that cut is on the right of $n$,

(d) or the part of $Q$ preceding $k$ is on the left of $n$, the part of $Q$ following $k$ is on the right of $n$, and the maximal entry $k$ of $Q$ coincides with $n$.

For instance, if $q=231$, then in $p=786923451$, the subsequence 786 precedes 9 , the subsequence 241 follows 9 , the subsequence 785 has its entries 7 and 8 before 9 and its entry 5 after 9 (corresponding to the cut 23|1), and the subsequence 895 starts before 9, uses 9, and ends after 9 .

3. If $q$ is decomposable and $q$ starts with its largest entry $k$, then

(a) either all of $Q$ is on the left of $n$,

(b) or all of $Q$ is on the right of $n$,

(c) or the part of $Q$ that precedes a given cut is on the left of $n$ and the part of $Q$ that follows that cut is on the right of $n$, or

(d) $Q$ starts with $n$, and the rest of $Q$ is on the right of $n$.

\section{Increasing Patterns}

Before proving that among all patterns of length $k$, it is the increasing pattern $12 \cdots k$ that occurs least often in 132-avoiding permutations, we prove a few general facts about the total number of increasing patterns in these permutations.

\subsection{A formula for increasing patterns}

Let $a_{n, k}$ be the total number of $12 \cdots k$-patterns in all $C_{n}$ permutations of length $n$ that avoid 132 . So for instance, $a_{2,1}=4, a_{3,1}=15$, and $a_{2,2}=1$.

Our goal in this subsection is to provide an explicit formula for the generating function $A_{k}(x)=$ $\sum_{n} a_{n, k} x^{n}$. We will use the well-known (see for instance Chapter 14 of [1]) explicit formula for the generating function of the Catalan numbers,

$$
C(x)=\sum_{n \geq 0} C_{n} x^{n}=\frac{1-\sqrt{1-4 x}}{2 x} .
$$

We will prove the following theorem.

Theorem 1. We have

$$
A_{1}(x)=\sum_{n \geq 1} n C_{n} x^{n}=\sum_{n \geq 1}\left(\begin{array}{c}
2 n \\
n
\end{array}\right) x^{n}-\sum_{n \geq 1} C_{n} x^{n}=\frac{1}{\sqrt{1-4 x}}-\frac{1-\sqrt{1-4 x}}{2 x} .
$$

Furthermore, for all positive integers $k \geq 2$, we have

$$
\begin{aligned}
A_{k}(x) & =A_{1}(x)\left(\frac{x C(x)}{1-2 x C(x)}\right)^{k-1}=A_{1}(x)\left(\frac{1}{2 \sqrt{1-4 x}}-\frac{1}{2}\right)^{k-1} \\
& =A_{1}(x) F^{k-1}(x) .
\end{aligned}
$$


Proof: For $k=1$, the claim is obvious, since an increasing subsequence of length one is just an entry of a permutation.

For larger $k$, an increasing subsequence of length $k$ is an indecomposable pattern. Hence the ways in which it can occur in the 132-avoiding permutation $p$ are listed in Case (1) of Fact 1 . This leads to the recurrence relations

$$
a_{n, k}=2 \sum_{i=1}^{n} a_{i-1, k} C_{n-i}+\sum_{i=1}^{n} a_{i-1, k-1} C_{n-i},
$$

or in terms of generating functions,

$$
\begin{aligned}
A_{k}(x) & =2 x A_{k}(x) C(x)+x A_{k-1}(x) C(x) \\
& =A_{k-1}(x) \frac{x C(x)}{1-2 x C(x)}
\end{aligned}
$$

and our claim follows by induction on $k$.

Note that in particular, 3 implies that for $1 \leq k<l$ we have

$$
A_{k}(x) A_{l}(x)=A_{k+1}(x) A_{l-1}(x) .
$$

\subsection{Why the Increasing Pattern is Minimal}

For a given pattern $q$, let $t_{n}(q)$ denote the number of all occurrences of the pattern $q$ in all 132-avoiding permutations of length $n$. So, in particular, if $q$ is the increasing pattern $12 \cdots k$, then $t_{n}(q)=a_{n, k}$.

The main result of this section is the following theorem, which shows that no pattern of a given length occurs less often in the set of 132-avoiding permutations than the increasing pattern of that length.

Theorem 2. Let $q$ be any pattern of length $k$. Then for all positive integers $n$, we have $t_{q}(n) \geq a_{n, k}$.

Before proving the theorem, we need to introduce some simple machinery to simplify notation.

Definition 1. Let $G(x)=\sum_{n \geq 0} g_{n} x^{n}$ and $H(x)=\sum_{n \geq 0} h_{n} x^{n}$ be two power series. We say that $G(x) \leq H(x)$ if $g_{n} \leq h_{n}$ for all $n \geq 0$.

Proposition 1. Let $G(x), H(x)$ and $W(x)$ be three power series with non-negative real coefficients so that $G(x) \leq H(x)$ holds. Then

$$
G(x) W(x) \leq H(x) W(x) .
$$

Proof: The coefficient of $x^{n}$ in $H(x) W(x)-G(x) W(x)$ is

$$
\sum_{i=0}^{n}\left(h_{i}-g_{i}\right) w_{n-i}
$$

which is a sum of non-negative real numbers, and is hence non-negative.

We can now return to the proof of Theorem 2

Proof of Theorem 2; We prove the statement by induction on $k$. For $k=1$, the statement is obvious.

Now let us assume that the statement is true for all positive integers less than $k$, and prove it for $k$. We distinguish three cases. Each of these cases will be handled by analyzing recurrence relations, which 
may sometimes seem somewhat cumbersome. Therefore, at the beginning of each case, we will give an intuitive description of that case.

In a permutation $p=p_{1} p_{2} \cdots p_{n}$, we say that $i$ is a descent if $p_{i}>p_{i+1}$. Otherwise, we say that $i$ is an ascent.

An overview of the cases is as follows. First, we treat patterns ending in their largest entry. Then we treat patterns that contain only one descent, say in position $j$. Finally, we treat all remaining patterns,

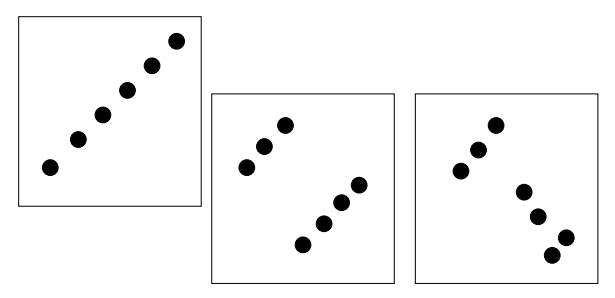

Fig. 2: Three types of patterns that we compare. comparing the patterns whose first descent is in position $j$ to the pattern whose only descent is in position $j$. See Figure 3.2 for an illustration.

Case 1: When $q$ ends in its largest entry $k$. Let $q^{\prime}$ be the pattern obtained from $q$ by removing the largest entry $k$ from the end of $q$. We then show that $q$ is more frequent than $12 \cdots k$ by showing that $q$ can be contained in a 132-avoiding permutation in the same ways as $12 \cdots k$, and then using the induction hypothesis. In other words, the difference between $a_{n, k}$ and $t_{n}(q)$ is caused by whatever happens before the last position of these patterns.

The possible ways in which $q$ can occur in a 132-avoiding permutation $p$ are listed in Case (1) of Fact 1 . Therefore, we have the recurrence relation

$$
t_{n}(q)=2 \sum_{i=1}^{n} t_{i-1}(q) C_{n-i}+\sum_{i=1}^{n} t_{i-1}\left(q^{\prime}\right) C_{n-i}
$$

leading to the generating function identities

$$
\begin{gathered}
T_{q}(x)=2 x C(x) T_{q}(x)+x C(x) T_{q^{\prime}}(x), \\
T_{q}(x)=T_{q^{\prime}}(x) \frac{x C(x)}{1-2 x C(x)} .
\end{gathered}
$$

Comparing formulae (4) and (7), we see that $T_{q}(x)$ is obtained from $T_{q^{\prime}}(x)$ by the same operation as $A_{k}(x)$ is obtained from $A_{k-1}(x)$, namely by a multiplication by the power series $F(x)=$ $\frac{x C(x)}{1-2 x C(x)}$. As

$$
\left[x^{n}\right] T_{q^{\prime}}(x)=t_{n}\left(q^{\prime}\right) \geq a_{n, k-1}=\left[x^{n}\right] A_{k-1}(x)
$$

by our induction hypothesis, and $F(x)=\sum_{n \geq 1}\left(\begin{array}{c}2 n-1 \\ n-1\end{array}\right) x^{n}$ has non-negative coefficients, our claim is immediate by Proposition 1 .

Case 2: Let us now consider the case in which $q$ does not end in its largest entry $k$, and the only descent of $q$ is in the position in which $k$ occurs. We will subsequently see that all remaining cases will easily reduce to this one. Let us say that $k$ is in the $j$ th position in $q$, with $j<k$. That is, $q=q_{k, j}=(k-j+1)(k-j+2) \cdots k 123(k-j)$. For instance, $q_{7,3}=5671234$. We will show that

$$
t_{n}\left(q_{k, j}\right) \geq t_{n}(12 \cdots k)=a_{n, k} .
$$


The main idea is the following. The pattern $q_{k, j}$ looks very similar to the increasing pattern, hence the ways in which $q_{k, j}$ can be contained in a 132-avoiding permutation are also similar to the ways in which the increasing pattern can. So the numbers $t_{n}\left(q_{k, j}\right)$ and $a_{n, k}$ satisfy very similar recurrence relations, and where they differ, they differ in the way we predicted.

Subcase 2.1: Let us assume first that $j \neq 1$, that is, that $k$ is not in the first position in $q_{k, j}$. As $q_{k, j}$ is decomposable by a cut after the $j$ th position, the ways in which $q_{k, j}$ can be contained in a 132-avoiding permutation are described in Case (2) of Fact 1 . That list leads to the recurrence relation

$$
\begin{aligned}
t_{n}\left(q_{k, j}\right)= & 2 \sum_{i=1}^{n} C_{i-1} t_{n-i}\left(q_{k, j}\right)+\sum_{i=1}^{n} a_{i-1, j} a_{n-i, k-j} \\
& +\sum_{i=1}^{n} a_{i-1, j-1} a_{n-i, k-j}
\end{aligned}
$$

and hence

$$
\begin{gathered}
T_{q_{k, j}}(x)=2 x C(x) T_{q_{k, j}}(x)+x A_{j}(x) A_{k-j}(x)+x A_{j-1}(x) A_{k-j}(x), \\
T_{q_{k, j}}(x)=\frac{x A_{k-j}(x)\left(A_{j}(x)+A_{j-1}(x)\right)}{1-2 x C(x)} .
\end{gathered}
$$

We need to show that $T_{q_{k, j}}(x) \geq A_{k}(x)$. Comparing formulae $(3)$ and (10), the preceding inequality is equivalent to

$$
\frac{x A_{k-j}(x)\left(A_{j}(x)+A_{j-1}(x)\right)}{1-2 x C(x)} \geq A_{1}(x) F(x)^{k-1},
$$

or

$$
\frac{x}{1-2 x C(x)} A_{1}(x)^{2}\left(F(x)^{k-3}+F(x)^{k-2}\right) \geq A_{1}(x) F(x)^{k-1} .
$$

Inequality (11) will be proved if we can show that

$$
\frac{x}{1-2 x C(x)} A_{1}(x)(1+F(x)) \geq F(x)^{2} .
$$

Indeed, 12 implies 11 by Proposition 1 , choosing $W(x)=F^{k-3}(x)$. On the other hand, (12) is equivalent to

$$
\begin{gathered}
\frac{x}{2(1-4 x)^{3 / 2}}+\frac{x}{2(1-4 x)}-\frac{1}{4(1-4 x)}+\frac{1}{4} \geq \frac{1}{4(1-4 x)}-\frac{1}{2 \sqrt{1-4 x}}+\frac{1}{4}, \\
\frac{x}{2(1-4 x)^{3 / 2}}+\frac{x-1}{2(1-4 x)}+\frac{1}{2 \sqrt{1-4 x}} \geq 0 .
\end{gathered}
$$

The coefficient of $x^{n}$ on the left-hand side is 0 if $n=0, n=1$, or $n=2$, and is $b_{n}=\left(\begin{array}{c}2 n-3 \\ n-2\end{array}\right)(2 n-1)-3 \cdot 2^{2 n-3}+\left(\begin{array}{c}2 n-1 \\ n-1\end{array}\right)$ if $n \geq 3$. If we replace $n$ by $n+1$, the negative 
summand in the above expression of $b_{n}$, that is, $3 \cdot 2^{2 n-3}$, grows fourfold, whereas a routine computation shows that the sum of the two positive terms grows $\frac{4 n^{2}+14 n+6}{n^{2}+3 n+2}$. fold. This fraction is larger than 4 for all $n \geq 3$, showing that $b_{n} \geq 0$ for all $n$, and our claim is proved.

Subcase 2, 2: If $j=1$, then a minor modification is necessary since if a copy of $q$ contains $n$, then it has to start with $n$. Hence formula 9 becomes

$$
\begin{aligned}
t_{n}\left(q_{k, 1}\right)= & \quad 2 \sum_{i=1}^{n} C_{i-1} t_{n-i}\left(q_{k, 1}\right)+\sum_{i=1}^{n} a_{i-1,1} a_{n-i, k-1} \\
& +\sum_{i=1}^{n} C_{i-1} a_{n-i, k-1} .
\end{aligned}
$$

So only the last sum is different from what it was in 97. This leads to the generating function identities

$$
\begin{gathered}
T_{q_{k, 1}}(x)=2 x C(x) T_{q_{k, 1}}(x)+x A_{k-1}(x)\left(A_{1}(x)+C(x)\right), \\
T_{q_{k, 1}}(x)=\frac{x A_{k-1}(x)\left(A_{1}(x)+C(x)\right)}{1-2 x C(x)} .
\end{gathered}
$$

Comparing formulae $\sqrt{4}$ and $\sqrt{13}$, the inequality $A_{k}(x) \leq T_{q_{k, 1}}(x)$ is now proved by Proposition 1, since $C(x) \leq A,(x)+C(x)$.

Case 3: Finally, there is the case when $k$ is in the $j$ th position of $q$ for some $j<k$, but $j$ is not the only descent of $q$. We claim that then copies of $q$ occur even more frequently than copies of $q_{k, j}$, roughly because even in segments where $q_{k, j}$ is increasing, $q$ is not.

That is, we will prove that

$$
t_{n}\left(q_{k, j}\right) \leq t_{n}(q)
$$

This, together with (8) will complete the proof of Theorem 2 .

As $q$ is decomposable by a cut after its $j$ th position, the ways in which $q$ can be contained in a 132-avoiding permutation are described in Case (2) of Fact 1 . Let $q^{<1>}$ denote the pattern formed by the first $j$ entries of $q$, and let $q^{<2>}$ be the pattern formed by the remaining $k-j$ entries of $q$. Then we have the recurrence relation

$$
t_{n}(q) \geq 2 \sum_{i=1}^{n} C_{i-1} t_{n-i}(q)+\sum_{i=1}^{n} t_{i-1}\left(q^{<1>}\right) t_{n-i}\left(q^{<2>}\right)+\sum_{i=1}^{n} t_{i-1}\left(q^{<1^{\prime}>}\right) t_{n-i}\left(q^{<2>}\right) .
$$

Here $q^{<1^{\prime}>}$ is the pattern obtained from $q^{<1>}$ by removing its last (and also largest) entry. Note that $t_{n}(q)$ is at least as large as the right-hand side, and not necessarily equal to it. That is because, unlike $q_{k, j}$, the pattern $q$ may be decomposable by other cuts, in addition to the cut after its $j$ th entry. The existence of such cuts would add extra summands to the right-hand side. 
The last displayed inequality leads to the generating function inequalities

$$
\begin{aligned}
T_{q}(x) & \geq 2 T_{q}(x) x C(x)+x T_{q^{<1>}>}(x) T_{q^{<2>}}(x)+x T_{q^{<1^{\prime}>}}(x) T_{q^{<2>}}(x), \\
T_{q}(x)(1-2 x C(x)) & \geq x T_{q^{1}}(x) T_{q^{2}}(x)+x T_{q^{<1^{\prime}}>}(x) T_{q^{<2>}}(x) .
\end{aligned}
$$

Note that as $1 /(1-2 x C(x))=1 / \sqrt{1-4 x}=\sum_{n \geq 0}\left(\begin{array}{c}2 n \\ n\end{array}\right) x^{n}$ has non-negative coefficients, the last displayed inequality remains true if we multiply both sides by $1 /(1-2 x C(x))$. This leads to the inequality

$$
T_{q}(x) \geq \frac{x T_{q<2>}(x)\left(T_{q<1>}+T_{q^{<1^{\prime}}>}(x)\right)}{1-2 x C(x)} .
$$

We can now compare formulae (10) and (15). We see that by our induction hypothesis, each factor on the right-hand side of (15) is at least as large as the corresponding factor on the right-hand side of 107 . That is, $x T_{q<2>}(x) \geq x A_{k-j}(x)$, since $q^{<2>}$ is a pattern of length $k-j$, and $T_{q<1>}+T_{q<1^{\prime}>}(x) \geq A_{k}(x)+A_{k-1}(x)$ by a summand-wise comparison. Hence, by Proposition 11. we have that $T_{q}(x) \geq T_{q_{k, j}}(x)$.

We have considered all cases, and proved our claim in each of them, hence the proof of Theorem 2 is complete.

\section{Decreasing Patterns}

In this section we prove that the decreasing pattern $k(k-1) \cdots 21$ occurs more frequently in 132-avoiding permutations than any other pattern of length $k$. The structure of the proof will be very similar to that of the minimality of the increasing pattern, but there will be more technical difficulties.

\subsection{General facts about decreasing patterns}

Let $d_{n, k}$ denote the number of decreasing subsequences of length $k$ in all 132-avoiding permutations of length $n$. Then we have

$$
d_{n, 1}=a_{n, 1}=n C_{n}=\frac{n}{n+1}\left(\begin{array}{c}
2 n \\
n
\end{array}\right) .
$$

For larger values of $k$, consider the set of all $C_{n}$ permutations of length $n$ that avoid 132. In that set, for every $1 \leq j \leq k-1$, there are

$$
\sum_{i=1}^{n} d_{i-1, j} d_{n-i, k-j}
$$

copies of $k(k-1) \cdots 1$ in which the first $j$ entries are on the left of $n$, and the last $k-j$ entries are on the right of $n$. (The index $i$ denotes the position of the entry $n$ in a permutation of length $n$.) In addition, there are $\sum_{i=1}^{n} C_{i-1} d_{n, k-1}$ copies of $k(k-1) \cdots 1$ that start with the entry $n$. Finally, there are the $2 \sum_{i=1}^{n} C_{i-1} d_{n-i, k}$ copies of $k(k-1) \cdots 1$ that are either entirely on the left of $n$, or entirely on the right of $n$. This leads to the recurrence relation

$$
d_{n, k}=\sum_{j=1}^{k-1} \sum_{i=1}^{n} d_{i-1, j} d_{n-i, k-j}+\sum_{i=1}^{n} C_{i-1} d_{n, k-1}+2 \sum_{i=1}^{n} C_{i-1} d_{n-i, k}
$$


and the generating function identities

$$
\begin{gathered}
D_{k}(x)=2 x C(x) D_{k}(x)+x C(x) D_{k-1}(x)+\sum_{j=1}^{k-1} x D_{j}(x) D_{k-j}(x), \\
D_{k}(x)=\frac{x C(x) D_{k-1}(x)+\sum_{j=1}^{k-1} x D_{j}(x) D_{k-j}(x)}{1-2 x C(x)} .
\end{gathered}
$$

The following corollary provides an estimate for the "growth" of the power series $D_{k}(x)$. It is worth comparing this result with Theorem 1 .

Corollary 1. We have

$$
D_{2}(x)=\frac{x D_{1}(x)}{(1-4 x)}
$$

and

$$
D_{k}(x) \geq \frac{x D_{k-1}(x)}{(1-4 x)}
$$

for $k \geq 3$.

Proof: The first displayed identity (the special case of $k=2$ ) immediately follows from (17) if we recall that $1-2 x C(x)=\sqrt{1-4 x}$ and that $D_{1}(x)+C(x)=\sum_{n \geq 0}\left(\begin{array}{c}2 n \\ n\end{array}\right) x^{n}=\frac{1}{\sqrt{1-4 x}}$.

The general formula (19) follows from (17) if we remove all summands from the right-hand side except for $x C(x) D_{k-1}(x)$ and $x D_{1}(x) D_{k-1}$, (we can do this since all the removed terms have non-negative coefficients), and then again, we recall that $D_{1}(x)+C(x)=\frac{1}{\sqrt{1-4 x}}$.

The following lemma is a natural counterpart of its much simpler analogue (6). It shows that the sequence of power series $D_{1}(x), D_{2}(x), \cdots$ is log-convex in a certain sense.

Lemma 1. For all positive integers $2 \leq a \leq b$ we have

$$
D_{a}(x) D_{b}(x) \leq D_{a-1}(x) D_{b+1}(x) .
$$

Proof: Induction on $a+b$. The smallest value of $a+b$ for which the statement is not trivial is 4 . The non-trivial statement then is that $D_{2}(x)^{2} \leq D_{1}(x) D_{3}(x)$. By $\left[18\right.$, this is equivalent to $D_{1}(x)^{2} \frac{x^{2}}{(1-4 x)^{2}} \leq$ $D_{1}(x) D_{3}(x)$. In order to prove the latter, it suffices to show that

$$
D_{1}(x) \frac{x^{2}}{(1-4 x)^{2}} \leq D_{3}(x)
$$

and that is immediate by $(19)$.

Now let us assume that the statement holds for $a+b=m-1$ and prove it for $a+b=m$. By (17), it suffices to show that

$$
D_{a}(x) C(x) D_{b-1}(x)+\sum_{j=1}^{b-1} D_{a}(x) D_{j}(x) D_{b-j}(x) \leq
$$




$$
\leq D_{a-1} C(x) D_{b}(x)+\sum_{j=1}^{b} D_{a-1}(x) D_{j}(x) D_{b+1-j}(x) .
$$

Note that the right-hand side has one more summand than the left-hand side. Now we carry out a series of pairwise comparisons. First, for the two terms preceding the summation signs, we have that $D_{a}(x) C(x) D_{b-1}(x) \leq D_{a-1}(x) C(x) D_{b}(x)$ since $C(x)$ has non-negative coefficients, and by our induction hypothesis, $D_{a}(x) D_{b-1}(x) \leq D_{a-1}(x) D_{b}(x)$. For the terms after the summation signs, for $j<a$, we have that

$$
D_{a}(x) D_{j}(x) D_{b-j}(x) \leq D_{a-1}(x) D_{j}(x) D_{b+1-j}(x)
$$

since our induction hypothesis implies that

$$
D_{a}(x) D_{b-j}(x) \leq D_{a-1}(x) D_{b+1-j}(x) .
$$

The induction hypothesis applies since $a+b-j<a+b$.

For $a \leq j \leq b-1$, we claim that

$$
D_{a}(x) D_{j}(x) D_{b-j}(x) \leq D_{a-1}(x) D_{j+1}(x) D_{b-j}(x) .
$$

(That is, we skip one summand, and we compare the $j$ th summand of the left-hand side to the $(j+1)$ st summand to the right-hand side.) Indeed, our induction hypothesis implies that

$$
D_{a}(x) D_{j}(x) \leq D_{a-1}(x) D_{j+1}(x) .
$$

The induction hypothesis applies since $a+j<a+b$.

Finally, we point out that each summand of the left-hand side was injectively associated to a weakly larger summand of the right-hand side. This proves our claim.

\subsection{Why the decreasing pattern is maximal}

Now we are in a position to state and prove the main result of this section.

Theorem 3. Let $q$ be a pattern of length $k$. Then the inequality

$$
t_{n}(q) \leq d_{n, k}
$$

holds.

Proof: We prove the statement by induction on $k$ and $n$. We know that the statement holds for $k=1$ and it is routine to verify it for $k=2$. Let us now assume that it is true for all patterns shorter than $k$. Let us further assume that for patterns of length $k$, the statement holds for all permutations shorter than $n$. (The initial cases of $n<k$ are obvious.) Let us now prove that the statement hold for permutations of length $n$, and patterns of length $k$.

Again, our proof proceeds by cases. We first handle patterns that start with their largest entry, then
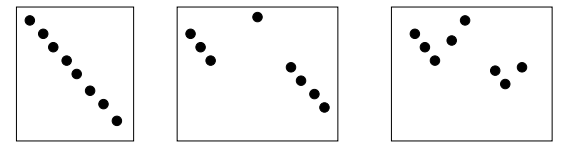

Fig. 3: Three kinds of patterns we compare. patterns which contain only one ascent, in position $j$. Finally, we cover the remaining cases, comparing patterns whose first ascent is in position $j$ to the pattern whose only ascent is in position $j$. See Figure 4.2 for an illustration of some of these cases. 
Case 1: Let us first consider the case when $q$ starts with its largest entry $k$. In this case, simply use the fact that the pattern obtained from $q$ by removing its first entry occurs less often than the decreasing pattern $k(k-1) \cdots 21$, and it is decomposable by no more cuts than $k(k-1) \cdots 21$.

In this case, $q$ is decomposable since it can be cut right after its first entry. Let us say that $q$ is decomposable with $v$ distinct cuts, and let $q^{1, f}$ and $q^{1, b}$ denote the patterns before and after the first cut, let $q^{2, f}$ and $q^{2, b}$ denote the patterns before and after the second cut, and so on, up to $q^{v, f}$ and $q^{v, b}$ for the patterns on the two sides of the last cut. (The letters $f$ and $b$ stand for "front" and "back".)

Note that the total length of $q^{j, f}$ and $q^{j, b}$ is always $k$ for every $j$, and that $\left|q^{1, f}\right|=1$ and $\left|q^{1, b}\right|=$ $k-1$.

Then we have the recurrence relation

$$
t_{n}(q)=\sum_{j=1}^{v} \sum_{i=1}^{n} t_{i-1}\left(q^{j, f}\right) t_{n-i}\left(q^{j, b}\right)+\sum_{i=1}^{n} C_{i-1} t_{n-i}\left(q^{1, b}\right)+2 \sum_{i=1}^{n} C_{i-1} t_{n-i}(q) .
$$

It is now straightforward to compare $d_{n, k}$ and $t_{n}(q)$ by comparing the corresponding summands of recurrence relations (16) and 20. Let us first compare the two double sums. In those sums, $j$ indexes the cuts of the respective patterns. As the decreasing pattern of length $k$ has $k-1$ cuts, $j$ ranges from 1 to $k-1$ in (16). In (20), $j$ ranges from 1 to $v$, where $v \leq k-1$ is the number of cuts that $q$ has. So the first double sum has more terms. We also claim that even the terms that the second double sum does have are smaller than the corresponding terms in the first double sum.

Indeed, if $\left|q^{j, f}\right|=y$ and $\left|q^{j, b}\right|=k-y$, then $t_{i-1}\left(q^{j, f}\right) \leq d_{i-1, y}$ and $t_{n-i}\left(q^{j, b}\right) \leq d_{n-i, k-y}$ by our induction hypothesis, so

$$
t_{i-1}\left(q^{j, f}\right) t_{n-i}\left(q^{j, b}\right) \leq d_{i-1, y} d_{n-i, k-y} .
$$

Comparing the second sums of (16) and 20 is even simpler. Their summands agree in the term $C_{i-1}$, and by our induction hypothesis, we know that $t_{n-i}\left(q^{1, b}\right) \leq d_{n-i, k-1}$. Finally, comparing the third sums of (16) and (20) we use the fact that $n-i<n$, so by our induction hypothesis, $t_{n-i}(q)<d_{n-i, k}$.

Case 2: $\quad$ When $q$ has only one ascent and $q$ does not start with its largest entry $k$. Just as in the previous section, this is the heart of the proof. The remaining cases will easily reduce to this one. Let $q^{k, h}$ be the pattern of length $k$ that avoids 132 and has only one ascent, in position $h$. As $q$ does not start in its largest entry $k$, this means that $k$ must be the $(h+1)$ st entry of $q$. That is,

$$
q^{k, h}=(k-1) \cdots(k-h) k(k-h-1) \cdots 321 .
$$

For instance $q^{5,2}=43521$.

The difficulty of this case is that there is a way in which $q^{k, h}$ can be contained in a 132-avoiding permutation in which $k(k-1) \cdots 21$ cannot, namely by having $h$ entries on the left of $n$ and $k-h-1$ entries on the right of $n$. As we will see, the ways in which the decreasing pattern can be 
contained in such a permutation and $q^{k, h}$ cannot are more prevalent, but that is not obvious. We will need Lemma 1 to prove that fact.

The structure of $q^{k, h}$ is somewhat similar to that of the decreasing pattern. That is, $q^{k, h}$ can be cut after each entry starting with $k$ (in position $h+1$ ). Cutting immediately after position $j$ (with $j \geq h+1$ ) will result in the two patterns $q^{j, h}$ and the decreasing pattern of length $k-j$. This leads to the recurrence relation

$$
t_{n}\left(q^{k, h}\right)=\sum_{j=h+1}^{k-1} \sum_{i=1}^{n} t_{i-1}\left(q^{j, h}\right) d_{n-i, k-j}+\sum_{i=1}^{n} d_{i-1, h} d_{n-i, k-h-1}+2 \sum_{i=1}^{n} C_{i-1} t_{n-i}\left(q^{k, h}\right) .
$$

We can carry out a pairwise comparison of corresponding sums in formulae (16) and (21). This is easiest for the third sums in those two formulae: indeed, as $n-i<n$, our induction hypothesis implies that $t_{n-i}\left(q^{k, h}\right) \leq d_{n-i, k}$ for all $k, i$, and $h$, so the third sum appearing in 16 is larger than the third sum appearing in 21].

As far as the double sums are concerned, for $j=h+1, h+2, \cdots, k-1$, our induction hypothesis implies that $t_{i-1}\left(q^{j, h}\right) \leq d_{i-1, j}$. Therefore, each term of the double sum of 21$]$ is at most as large as the corresponding term of (16).

Therefore, the claim $t_{n}\left(q^{k, h}\right) \leq d_{n, k}$ will be proved if we can show that the remaining sum in 21p is less than the remaining sums in [16], that is, that

$$
\sum_{i=1}^{n} d_{i-1, h} d_{n-i, k-h-1} \leq \sum_{j=1}^{h} \sum_{i=1}^{n} d_{j, i-1} d_{n-i, k-j}+\sum_{i=1}^{n} C_{i-1} d_{n-i, k-1} .
$$

We show the stronger statement that the above inequality remains true even if we remove all summands from the double sum in which $j \neq 1$. (Note that as $k$ is not in the first position of $q$, we know that $h-1 \geq 1$, so this will leave a non-empty set of summands.) In other words, we claim that

$$
\sum_{i=1}^{n} d_{i-1, h} d_{n-i, k-h-1} \leq \sum_{i=1}^{n} d_{i-1,1} d_{n-i, k-1}+\sum_{i=1}^{n} C_{i-1} d_{n, k-1} .
$$

This is equivalent to the generating function inequalities

$$
\begin{gathered}
x D_{h}(x) D_{k-h-1}(x) \leq x D_{1}(x) D_{k-1}(x)+x C(x) D_{k-1}(x) . \\
D_{h}(x) D_{k-h-1}(x) \leq D_{k-1}(x)(1-4 x)^{-1 / 2} .
\end{gathered}
$$

By Lemma 1, we know that $D_{h}(x) D_{k-h-1}(x) \leq D_{1}(x) D_{k-2}(x)$, so it suffices to prove that

$$
D_{1}(x) D_{k-2}(x) \leq D_{k-1}(x)(1-4 x)^{-1 / 2} \text {. }
$$


By Corollary 1 , we know that $\frac{x}{1-4 x} D_{k-2}(x) \leq D_{k-1}(x)$, so our claim will be proved if we can show that

$$
D_{1}(x) \leq \frac{x}{(1-4 x)^{3 / 2}} .
$$

The last displayed inequality holds since $\left[x^{n}\right] D_{1}(x)=\frac{n}{n+1}\left(\begin{array}{c}2 n \\ n\end{array}\right)$ whereas $\left[x^{n}\right] \frac{x}{(1-4 x)^{3 / 2}}=(2 n-$ 1) $\left(\begin{array}{c}2 n-2 \\ n-1\end{array}\right)$. A routine computation shows that the latter is larger as soon as $2<n+1$, or $1<n$.

Case 3: Finally, we consider the case when $q$ has more than one ascent, and $q$ does not start with its maximal entry. Let us assume that the maximal entry $k$ of $q$ is in the $h$ th position. We will show that then $t_{n}(q) \leq t_{n}\left(q^{k, h}\right)$. The main idea behind the proof is that $q^{k, h}$ is decomposable at every place where $q$ is, and after decomposition, its parts are close to the decreasing pattern.

Let us inductively assume that we know the statement $t_{n}(q) \leq t_{n}\left(q^{k, h}\right)$ for all patterns shorter than $k$.

The set of positions after which $q$ can be cut is a subset of the set of positions after which $q^{k, h}$ can be cut. Just as in Case (1), let us say that $q$ is decomposable with $v$ distinct cuts, and let $q^{1, f}$ and $q^{1, b}$ denote the patterns before and after the first cut, let $q^{2, f}$ and $q^{2, b}$ denote the patterns before and after the second cut, and so on, up to $q^{v, f}$ and $q^{v, b}$ for the patterns on the two sides of the last cut. Note that $\left|q^{1, f}\right|=h$ and $\left|q^{1, b}\right|=k-h$.

Then, similarly to Case (1), we have the recurrence relation

$$
t_{n}(q)=\sum_{j=1}^{v} \sum_{i=1}^{n} t_{i-1}\left(q^{j, f}\right) t_{n-i}\left(q^{j, b}\right)+\sum_{i=1}^{n} t_{i-1}\left(q^{1, f^{\prime}}\right) t_{n-i}\left(q^{1, b}\right)+2 \sum_{i=1}^{n} C_{i-1} t_{n-i}(q),
$$

where $q^{1, f^{\prime}}$ is the pattern $q^{1, f}$ with its last (and largest) entry removed.

The inequality $t_{n}(q) \leq t_{n}\left(q^{k, h}\right)$ is now obvious by pairwise comparing the three summands in (21) and 22), and using the induction hypotheses. (In particular, when comparing the first summands, we use the fact that if $\left|q^{j, h}\right|=\left|q^{j, f}\right|$, then $t_{i-1}\left(q^{j, h}\right) \geq t_{i-1}\left(q^{j, h}\right)$ by the induction hypothesis made in this case. Recall that we assumed that for patterns $q$ shorter than $k$, it is true that $t_{n}(q) \leq t_{n}\left(q^{|q|, h}\right)$, where $h$ is the first ascent of $q$.)

\section{Asymptotic Enumeration}

Theorem 1 provides an explicit formula for $A_{2}(x)$. ¿From that formula, it is routine to deduce that

$$
a_{n, 2}=2^{2 n-1}-\frac{1}{4}\left(\begin{array}{c}
2 n+2 \\
n+1
\end{array}\right)-\frac{n}{2 n+1}\left(\begin{array}{c}
2 n \\
n
\end{array}\right)
$$

for $n \geq 1$.

Similarly, an explicit formula for $D_{2}(x)$ can be obtained by setting $k=2$ in 17 . That is,

$$
D_{2}(x)=\frac{x C(x) D_{1}(x)+x D_{1}(x) D_{1}(x)}{1-2 x C(x)}=\frac{x}{(1-4 x)^{3 / 2}}-\frac{1}{2(1-4 x)}+\frac{1}{2 \sqrt{1-4 x}} .
$$


This yields

$$
d_{n, 2}=\left(\begin{array}{c}
2 n-1 \\
n-1
\end{array}\right)(n+1)-2^{2 n-1}
$$

for $n=1$.

Comparing formulae (23) and (25) and using Stirling's formula, we see that in 132-avoiding permutatations of length $n$, there are $c \sqrt{n}$ times as many inversions as non-inversions.

\section{Further Directions}

A simple analysis of the proofs of Theorems 2 and 3 shows that the inequalities $a(n, k) \leq t_{n}(q) \leq d(n, k)$ are sharp if $n$ is large enough compared to $k$.

We have seen that, if we consider 132-avoiding permutations, then among all patterns of length $k$, the increasing pattern is the least likely to occur and the decreasing pattern is the most likely to occur. This suggests the following natural question.

Question 1. Let $r$ be any pattern, and let $t_{r, q}(n)$ be the number of all copies of $q$ in all r-avoiding permutations of length $n$. Let us assume that among all patterns $q$ of length $k$, it is the increasing pattern that minimizes $t_{r, q}(n)$ for all $n$.

Is it then true that among all patterns $q$ of length $k$, it is the decreasing pattern that maximizes $t_{r, q}(n)$ ?

Another direction of research is the following.

Question 2. Let $q_{1}$ and $q_{2}$ be two patterns of the same length, and assume that for some positive integer $N$, the inequality

$$
t_{r, q_{1}}(N)<t_{r, q_{2}}(N)
$$

holds. Is it then true that

$$
t_{r, q_{1}}(n)<t_{r, q_{2}}(n)
$$

for all $n>N$ ?

In other words, is it true that the relation between the frequency of $q_{1}$ and $q_{2}$ in $r$-avoiding permutations depends only on $r, q_{1}$ and $q_{2}$, or does it depend on $n$ as well?

Lemma 1 and formula 6 show interesting combinatorial properties of the power series $D_{1}(x), D_{2}(x), \cdots$ and $A_{1}(x), A_{2}(x), \cdots$. These properties are easy to express in terms of combinatorial objects, without power series. However, is there a combinatorial proof for them?

\section{References}

[1] M. Bóna, A Walk Through Combinatorics, 2nd edition, World Scientific, 2006.

[2] M. Bóna, Combinatorics of Permutations, CRC Press, 2004.

[3] M. Bóna, Where the monotone pattern (mostly) rules, Discrete Math. 308 (2008), no. 23, 5782-5788.

[4] J. Cooper, Combinatorial Problems I like, internet resource, http://www.math.sc.edu/\%7Ecooper/combprob.html

[5] N. Ruskuc, S. Linton, V. Vatter, editors, Patterns in Permutations, Cambridge University Press, 2010.

[6] R. Stanley, Enumerative Combinatorics, Volume 2, Cambridge University Press, 1997. 